Objectives The objectives of this study were to 1 . Determine occurrence of iron and vitamin D deficiency in the sample population, 2. Identify predictors of the presence of these deficiencies if any and 3. Elucidate factors influencing screening for these deficiencies in children with ASD.

Methods This was a retrospective cross-sectional review of case records of all patients with ASD who were seen at a tertiary developmental paediatric centre in Singapore from January 2018 to June 2018. Inclusion criteria was 1. Child age 0 to 7 years and 2. Diagnosis of ASD following clinical evaluation by a developmental paediatrician or formal psychological evaluation with the Autism Diagnostic Observation Schedule. Exclusion criteria was 1. Chronic medical conditions and 2. Genetic syndromes. Information on demographic variables, ASD-related variables and other medical conditions was abstracted using a structured data collection form. Presence of iron deficiency (serum ferritin $<12 \mu \mathrm{g} / \mathrm{L}$ or transferrin saturations $<10 \%)$, vitamin D deficiency $(25$-hydroxyvitamin D $[25$ $(\mathrm{OH}) \mathrm{D}]<10 \mu \mathrm{g} / \mathrm{L})$ and vitamin $\mathrm{D}$ insufficiency $(25(\mathrm{OH}) \mathrm{D}$ between $10.1-29.9 \mu \mathrm{g} / \mathrm{L}$ ) was determined from review of laboratory investigations. Descriptive statistics were used to assess for prevalence of iron and vitamin D deficiencies. Logistic regression was used to identify predictors of iron and vitamin $\mathrm{D}$ deficiencies and chi square tests were used to compare children who were and were not offered screening for these deficiencies.

Results The sample consisted of 480 children (81\% males, $19 \%$ females) with a mean age of 4.5 years (SD 1.3). Of this, only $20.2 \%(\mathrm{~N}=97)$ of children were screened for iron deficiency and $18.3 \%(\mathrm{~N}=88)$ were screened for vitamin $\mathrm{D}$ deficiency using blood tests. The prevalence of iron deficiency was $19 \%(\mathrm{~N}=18)$. Younger children were more likely to have iron deficiency $(\mathrm{B}=1.06, \mathrm{p}=0.02)$. The prevalence of vitamin $D$ insufficiency was $38 \%(\mathrm{~N}=33)$ and that of vitamin $\mathrm{D}$ deficiency was $1.1 \%(\mathrm{~N}=1)$. Older children were more likely to have vitamin $\mathrm{D}$ deficiency or insufficiency $(\mathrm{B}=1.07, \mathrm{p}=0.01)$. Only $20.8 \% \quad(\mathrm{~N}=100)$ of children from the entire sample were offered screening blood tests; children with greater severity of disease $(\chi 2=9.80, \mathrm{p}=0.002)$ and those with a history of selective feeding $(\chi 2=8.27, \mathrm{p}=0.004)$ were more likely to be offered screening tests.

Conclusions The prevalence of both iron deficiency and vitamin D deficiency/insufficiency was high in this sample compared to the general population. Of note, only a small proportion of children were offered screening and eventually screened for these deficiencies. Apart from child age, there were no significant child or disease characteristics that predicted these deficiencies. Routine screening of all children with ASD for iron and vitamin D deficiency is recommended.

\section{FRACTURE BURDEN IN PAEDIATRIC END STAGE KIDNEY DISEASE}

Samuel Chung-sum Ho, Eugene Yu-hin Chan, Ellen Yu, Matthew Hon-lam Lee, Yuetling Tung, Alison Lap-tak Ma. Hong Kong

\subsection{6/bmjpo-2021-RCPCH. 185}

Background Paediatric patients with chronic kidney disease are known to have an increased risk of fracture. However, data pertaining to children with end stage kidney disease (ESKD) receiving renal replacement therapy (RRT) is limited.
Objectives The aim of this study is to determine the incidence of fracture and associated factors in this specific group of patients.

Methods We conducted a retrospective review on all paediatric patients with ESKD at the tertiary Paediatric Nephrology Centre in Hong Kong. Children who presented before 18 years with active follow-ups for 12 or more months by November 2020 were included.

Results RRT was initiated in 69 children $(55 \%$ boys) at a mean age of $9.2 \pm 5.9$ years. At the time of evaluation, 21 (30.4\%), $10(14.5 \%)$ and $38(55.1 \%)$ patients received peritoneal dialysis (PD), haemodialysis (HD) and kidney transplant respectively. 3 patients $(4.3 \%)$ had prior kidney graft loss and resumed on dialysis. One patient (1.5\%) reported a fracture prior to RRT.

Over a median of 5.2 years (IQR 3.0-8.3) follow-up, 10 fracture episodes were observed in 7 patients $(10.1 \%)$ at a mean duration of $7.8 \pm 8$ years since RRT initiation, corresponding to a cumulative fracture incidence of 227.8 per 10000 patient year (95\% CI, 86.6-369.0). This rate was 5folds higher than published data from our local general paediatric population (45 per 10,000 person-years; 95\% CI, 43.9$46.1 ; \mathrm{p}=0.01)$. Of note, all patients experienced single fracture episode except one child who developed 4 fracture episodes.

Children who sustained fractures were significantly younger at the time of RRT initiation ( 3.5 vs 10.4 years; $p=0.02)$ and had a longer time on dialysis (12 vs 2.7 years; $\mathrm{p}<0.001)$. Other factors associated with fractures included metabolic bone disease $(28.6 \%$ versus $1.6 \%$; $=0.03)$, difficulty in walking $(28.6 \%$ versus $3.2 \% ; \mathrm{p}=0.05)$, radiological evidence of renal osteodystrophy $(85.7 \%$ vs $25.8 \% ; \mathrm{p}=0.003)$, parathyroid hyperplasia/adenoma $(100 \%$ vs $31.8 \% ; \mathrm{p}=0.01)$ and a higher parathyroid hormone level (pmol/L) (62.7 vs 30.3; p=0.02). Calcium, phosphate and ALP levels, as well as the proportion of patients with vitamin D deficiency were similar between the two groups. While the practice of native and active vitamin $\mathrm{D}$ and phosphate binders were not different, more patients with fracture received cinacalcet $(57.1 \%$ versus $12.9 \%$; $=0.02)$, which may suggest more severe hyperparathyroidism.

Conclusions Children with ESKD receiving RRT are at a higher risk of fracture. Longer duration of dialysis and a higher average parathyroid hormone level were potential modifiable factors associated with fractures.

\section{INTEGRATED LEARNING THROUGH SIMULATIONS WITH THE AVIATION INDUSTRY}

Kee Wei Phang, Poonam Patel. UK

\subsection{6/bmjpo-2021-RCPCH.186}

Background The aviation industry has been the leader in the field of simulation having pioneered and designed much of their current simulation training including crew resource management, situational awareness and debriefing tools. Increasingly, there is an interest in incorporating key aspects of such simulation training into the medical teaching curriculum as many of the skills, particularly with regards to human factors are transferrable skills to medicine. With this in mind and the given the current COVID pandemic which has rendered much of the aviation industry to a halt, the simulation faculty at 
North Middlesex University Hospital developed the first joint simulation programme with some of the British Airways pilots (Project Wingman) locally.

Objectives The objectives of the joint simulation programme is to develop and improve human factors training amongst staff working the Emergency Department, by tapping on the expertise of the aviation crew in delivering key situation awareness and crisis management skills, integrating it within the relevant clinical environment so as to enhance patient care.

Methods The simulation faculty developed a clinical scenario relevant for the trainees and delivered the simulation in situ (ie within the emergency department) whilst the BA pilots who were briefed on the clinical aims of the simulation beforehand, observed the simulation and provided verbal and written feedback following the simulation. The written feedback with relatable human factor references to the aviation industry was produced but the pilot within a week following the simulation, which enabled learners continue the reflective process after the simulation at their own pace. In addition, pre and post simulation feedback was collected from the participants to better understand and improve further delivery of these simulations training events.

Results To date, Project Wingman has conducted 2 in situ simulations in the North Middlesex University Hospital with plans for further simulation dates. Attendance of the simulation involved the core simulation faculty team, BA pilots and a multidisciplinary team of clinical staff including emergency physicians, anaesthetists, nurses and operating department practitioners. Feedback from these simulations have been overwhelmingly positive, with over $90 \%$ of the participants finding it relevant and useful for their training. There is also a shift in attitude as demonstrated in the pre and post simulation feedback where hesitancy amongst participants towards the usefulness of incorporating aviation standards for providing feedback reduced.

Conclusions In conclusion, the North Middlesex University Hospital simulation faculty feel that these joint simulation programmes are beneficial to the emergency department and provides a fresh perspective towards human factors training as well as situational awareness and debriefing methods. The skills learnt from such simulation training enhances the team's ability to work cohesively as a multidisciplinary team even during highly stressful situations. Moreover, the provision of detailed written feedback following each session allows the learner to continue reflecting on the event and for the wider team who could not attend the session learn from it as well. A combined simulation programme will undoubtedly help clinicians to continue developing their skills in delivering the highest standard of patient care through challenging situations.

\section{SAFEGUARDING NON-MOBILE BABIES IN THE EMERGENCY DEPARTMENT}

Laura Duthie, Amanda King, Will Christian, Ross Goodson, Carmel Rosin, Abi Tyer. UK

10.1136/bmjpo-2021-RCPCH. 187

Background Accidental injuries in non-mobile babies are very uncommon, therefore even minor injuries could indicate serious abuse and require appropriate investigation.

In our ED in a large tertiary children's hospital in the UK, any non-mobile baby presenting with a visible injury is considered at risk of abuse and therefore follows a standardised pathway. This involves a multi-agency approach in which checks are conducted with social care and the police to ensure the clinician can make an informed decision about the safety of the child. However, audits in 2016 and 2017 demonstrated that compliance to the pathway was poor $(68.6 \%)$ with only half of clinicians completing a background check $(54.3 \%)$ or passing on these results to the GP (51.4\%).

Objectives Our aim was to identify obstacles to the non mobile baby pathway and address them in order to streamline the process for clinicians, social care and families.

Methods A multidisciplinary task group was set up including members from clinical and administration teams. With the assistance of our QI department, we completed process mapping, fishbone analysis and a prioritisation matrix. A questionnaire was then distributed amongst clinicians in order to identify specific obstacles to the pathway, and the results analysed.

Results The questionnaire was completed over two weeks in November 2020 by 27 of the medical and nurse practitioner team ranging in experience from 3 months to over 10 years.

Results identified that although all clinicians were aware of the process and $93 \%$ were able to identify patients who needed to undergo it, only $78 \%$ were confident in actually undertaking the process.

The time taken to complete the process ranged from 10 to 120 minutes amongst participants and of those who provided numerical responses, over half stated it took over 60 minutes.

Half (48\%) of respondents found it difficult to locate the phone number to call when undertaking the checks.

When asked for areas of improvement within the process, participants most commonly identified; the need for a clear guideline, easy access to phone numbers, leaflets for parents and further training for clinicians and social care.

Conclusions We believe that our process for managing injuries in non-mobile babies is fairly unique and has already improved identification of children at risk of significant harm. The feedback gained and subsequent analysis has enabled us to recognise and address specific obstacles which can be targeted for improvement, to ensure a more streamlined process that it is both time efficient and as safe as possible.

A simplified guideline and patient information leaflet has been created and approved, along with a poster for our waiting room to explain the procedure to families. We also continue to improve awareness and understanding of the procedure amongst our colleagues through education. In the near future, we also hope to integrate the procedure within our IT infrastructure to allow a more efficient pathway.

We believe that our changes enable significant improvement of the non-mobile baby pathway for both clinicians and families, in what can be a challenging and stressful process.

\section{6 EVALUATING THE SUCCESS RATES OF RADIOLOGICALLY INSERTED GASTROSTOMY, GASTROJEJUNOSTOMY AND JEJUNOSTOMIES IN PAEDIATRIC PATIENTS AT ST. GEORGE'S HOSPITAL}

Aditya Mavinkurve. UK

\subsection{6/bmjpo-2021-RCPCH.188}

Background In the past, gastrostomy tubes had to be inserted laparoscopically, however recently the trend has shifted to favour the use of percutaneous endoscopic gastrostomies, 